\title{
Factibilidad de una Microempresa de Servicios de apoyo a Productores Pequeños de Nuez Pecanera
}

Feasibility of a Micro-Enterprise Of Support Services To Small Producers of Nut Pecan

Magaña Magaña José Eduardo ${ }^{\natural}$, Villarreal Ramírez Víctor Hugo ${ }^{1}$, González Anchondo María Elvira², Kiessling Davison Christian Mauricio ${ }^{1}$, Palacios Monarrez Abdón ${ }^{1}$

${ }^{1}$ Profesor de la Facultad Ciencias Agrícolas y Forestales de la Universidad Autónoma de Chihuahua, km 2.5 carretera Delicias-Rosales. ${ }^{2}$ profesores de la Facultad de Contaduría y Administración de la Universidad Autónoma de Chihuahua.

${ }^{凶}$ Autor para correspondencia: emagana@uach.mx

Recibido: $15 / 09 / 2018$

Aceptado: $15 / 11 / 2018$

\section{RESUMEN}

En nuestra región no existe una empresa de servicios de recolección, limpia y selección en la unidad productora de nuez, que sea móvil con maquinaria especializada. Estos servicios permiten a los productores obtener un precio mejor de su producto, logrando así una ganancia mayor a la hora de vender su nuez. Por esta razón, se decidió analizar la factibilidad de la puesta en marcha de una empresa de servicios de recolección, limpia y selección de nuez con el uso de maquinaria especializada, que le brindará al productor ventajas competitivas. Se condujo una investigación de mercado con el objetivo de conocer preferencias de los productores y datos relacionados con la realización y puesta en marcha de la empresa. La evaluación del proyecto se realizó para evaluar la factibilidad tomando en cuenta que el costo del proyecto es de $\$ 866,199.84$, el cual tiene un horizonte de 5 años. El indicador de evaluación de la Tasa Interna de Retorno (TIR) nos dio como resultado 27.76\%, el indicador del Valor Actual Neto (VAN) nos dio como resultado $\$ 88,460$. Ambos indicadores nos indican que el proyecto es viable desde el punto de vista financiero y económico. Desde el punto de vista del mercado, el $86 \%$ de los encuestados, productores de nuez de hasta 5 ha, manifestaron su aceptación de utilizar el servicio de la empresa.

Palabras clave: Tecnología, productor de nuez, estudio de mercado, rentabilidad de la empresa.

\begin{abstract}
In our region there is not a company that collect, cleans, and selection nut-pecan to small producers of nut pecan with specialized machinery. These services allow producers to get a better price for their product, thus achieving one greater profit when selling her nut. For this reason, it was decided to analyze the feasibility of starting up a company of services of clean, collection, selection of walnut with the use of specialized machinery, which will give the producer competitive advantages. Led market research in order to meet preferences of producers and data related to the realization and implementation underway of the company. The evaluation of the project was to evaluate the
\end{abstract}


feasibility, taking into account that the cost of the project is $\$ 866,199.84$, which has a 5 -year horizon. Evaluation of the internal rate of return (IRR) prompt us resulted $27.76 \%$, the indicator of the net present value (VAN) gave us as a result $\$ 88,460$. Both indicators show that the project is viable from the economic and financial point of view. From the point of view of the market, $86 \%$ of respondents, producing up to 5 Walnut ha, expressed acceptance to use the service of the company.

Keywords: technology, service to the producer of walnut, market study, profitability of the company.

\section{INTRODUCCION}

La producción de nuez pecanera en México es de aproximadamente 79 mil toneladas por año, de las cuales 54 mil se producen en el estado de Chihuahua como principal productor, otros estados que destacan en su producción es Coahuila, la Comarca Lagunera (Coahuila-Durango), Sonora y Nuevo León, el rendimiento promedio de este cultivo es de 1.6 ton/ha (SIAP- SAGARPA, 2016). En nuestra región se tiene la apreciación de que no existe una microempresa que proporcione servicios de recolección, limpia y selección de nuez pecanera para productores de 1 a 5 has, que sea móvil y con maquinaria especializada. México es el segundo productor de nuez a nivel mundial, con una superficie plantada de 59,000 ha,

Definición Del Problema ¿Es factible la realización de una empresa dedicada a prestar servicios de recolección, limpieza y selección de nuez pecanera utilizando tecnología?

Por esta razón, se decidió analizar la factibilidad de la puesta en marcha de una empresa de servicios de recolección, limpia y selección de nuez con el uso de maquinaria especializada, que le brindará al productor ciertas ventajas competitivas para optimizar el tiempo de recolección, limpieza y selección. Así como optimizar los recursos económicos del productor en relación a gastos de vigilancia y robo hormiga del cultivo y abre posibilidades a productores de exportar a mercados exigentes.

El objetivo general fue realizar un estudio de factibilidad de una microempresa que brinde el servicio de recolección, limpieza y selección de nuez en el sitio de producción. En tanto que los objetivos específicos fueron realizar un estudio de factibilidad y analizar la información para determinar el su posible puesta en marcha.

La agricultura ha cambiado en los últimos 50 años. Este cambio en el uso de la fuerza ha modificado los procedimientos agrícolas. Usamos diferentes máquinas y nuevos métodos de producción. Plantamos diferentes cultivos y obtenemos mayores rendimientos. Invertimos más en nuestras máquinas agrícolas y utilizamos a nuestros trabajadores de manera diferente, más eficiente. Nuestras operaciones agrícolas son diferentes; disponemos de los campos con un criterio diferente; empleamos nuestro tiempo de forma diferente.

Los tractores y maquinas más rápidas, de mayor capacidad y de mayor compilación exigen mejores materiales, mejor proyecto de ingeniería, mejor servicio de reparaciones y 
mantenimiento, puntualidad en su operación en el campo, y un manejo eficaz. Ante todo, exigen una selección cuidadosa y una operación diestra y mantenimiento correcto (Stone \& Gulvin, 1982).

Se promueve la constitución de empresas con una orientación clara a los mercados y demandas específicas que utilicen insumos y procesos con una mayor eficiencia económica. Sabiendo que los productores poseen un gran conocimiento de sus procesos productivos, y que el desarrollo de nuevas alternativas sólo puede alcanzarse a partir de las condiciones, los conocimientos, intereses de los productores, la forma en que puede impulsarse ese proceso es a través del desarrollo de empresas de mayor capacidad económica mediante la asociación de productores para realizar algunas actividades específicas, como la venta de productos, la compra de insumos o la venta de un servicio especializado (Fuentes, 2007).

\section{MATERIALES Y MÉTODOS}

El siguiente trabajo se realizó teniendo en cuenta la metodología SAGARPA para la elaboración de proyectos de inversión. El diseño de la investigación será exploratoria ya que va a proporcionar conocimiento y comprensión del problema, así como también de carácter conclusiva puesto que ayuda a guiar la toma de sediciones y seleccionar el mejor camino de la investigación, descriptiva puesto que se requieren describir las características de compra de los productores locales de nuez y las funciones del mercado actual de nuestros competidores: precios, tiempo de brindar el servicio por kilogramo, y otros. Se realizaron pláticas con los encargados actuales de las seleccionadoras, los encargados de brindar el servicio de recolección y los productores locales de nuez con el objetivo de recolectar información importante relativa al servicio.

Se realizaran entrevistas a productores locales de nuez de la región en Meoqui, con el objetivo de conocer datos de interés para el proyecto como:

Como datos ya conocidos es útil conocer que el precio de recolección dela nuez utilizando el vibrador es de 70.00 pesos por árbol, la selección que en su mayoría se realiza manual, contratando gente se paga de 3 a 4 pesos por kilogramo y una persona puede seleccionar hasta $100 \mathrm{~kg}$. Por día. El precio al que compran la nuez varia. Nuez de campo la compran en 25.00 A 30.00 por kilogramo y la nuez seleccionada de 35 a 60 pesos por kilogramo dependiendo de la calidad del producto y la seleccionadora. Si se decide seleccionar el producto en alguna de las seleccionadoras de la región el precio por kilogramo varia de 2 a 3 pesos por kilogramo. La realización de una encuesta, que se llevara a cabo a productores locales, dará a conocer aspectos relevantes en cuanto a los servicios que ellos utilizan y el costo, así como la duración de los mismos, lo cual permitirá a nuestra empresa poder posicionarse con las ventajas competitivas que posee en cuestión de optimización de recurso tiempo y dinero. Por lo que el problema de investigación se centra en el posicionamiento de la empresa en la región, principalmente dedicado a los productores locales.

Dividiremos los posibles productores por tamaño de superficie, luego se aplicaran los cuestionarios, se recolecta la información en 
el programa estadístico SPSS y se presentan los resultados obtenidos y conclusiones.

Se aplicó un cuestionario a productores pequeños de hasta 10 ha de la región de Meoqui y se visitaron seleccionadoras y empresas que brinden el servicio de recolección para obtener mayores datos. El

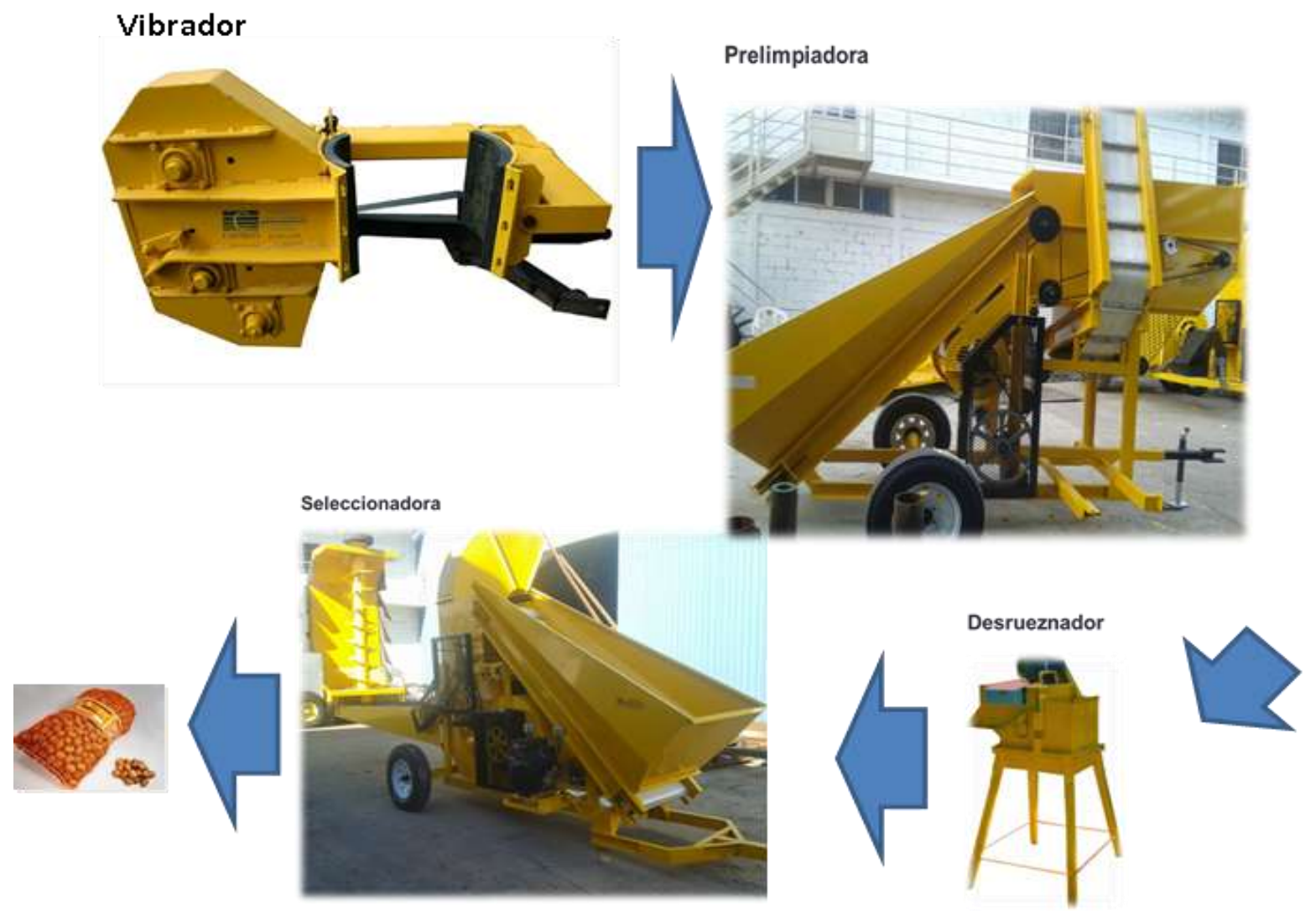

tamaño de la muestra tiene que ver con el número total de pequeños $\mathrm{y}$ medianos productores que hay en la región. El cuestionario se realiza de forma personal con cada uno de los productores locales.

Proceso de recolección, limpieza y selección de nuez y la maquinaria necesaria.
Vibrador modelo 7000 La parte inicial del servicio comienza con la estación 1: Recolección del cultivo, 3 operarios realizarán la colocación de mallas alrededor de los árboles, con el objetivo de facilitar el transporte del producto a la estación 2 . Un técnico de maquinaria utilizara el vibrador modelo 7000 para nogales de hasta $60 \mathrm{~cm}$. de diámetro con toma de fuerza y control remoto hidráulico para enganche universal de tres puntos para cosechar el producto del árbol para posteriormente pasar a la estación 3 .
Prelimpiadora dos operarios trasladan el material contenido en las lonas a la maquina pre limpiadora de jalón con llantas accionada con toma de fuerza, la cual consta de una tolva elevadora, una criba de 28 pulgadas de ancho con aire, depuradora de palos y hojas con banda de descarga posteriormente se conecta a un remolque, recolectando en éste toda la nuez con ruezno limpia para después pasar a la estación 4.

Desrueznador El proceso siguiente es la estación 4, un operario inclina el remolque 
especial para trasladar el producto hacia el desrueznador de 8 pulgadas, el cual consta de un motor de 3 H.P. trifásico esta máquina es la encargada de retirar el ruezno de la nuez. Esta máquina puede procesar una tonelada en 1 hora. De aquí pasa a un contenedor especial (anexo 4) para posteriormente pasar a la estación 5. Ver foto (anexo 5).

Seleccionadora $\mathrm{El}$ proceso final es la selección del cultivo en la estación 5 un operario es el encargado de transportar el contenedor especial que contiene la nuez limpia y sin ruezno a la máquina seleccionadora de nuez, la cual consta de un jalón con llantas que contiene un elevador, un soplador de turbina eficiente para nuez vana con mesa de selección de tres carriles con encostaladores al final, para diferenciación de producto, accionada con motores eléctricos de 10 y 5 H.p. Aquí el producto es puesto en arpillas de forma automática, para después ser etiquetado por un operario y revisado por el ingeniero de campo en aspectos de calidad, como empaque y limpieza del producto terminado.

.Cuadro 1. Conceptos de inversión de la empresa EL NOGAL,

\begin{tabular}{|c|c|c|c|c|c|c|}
\hline \multirow[b]{2}{*}{ Concepto } & \multirow[b]{2}{*}{ Unidad } & \multirow[b]{2}{*}{ Cantidad } & \multirow[b]{2}{*}{ P. Unitario } & \multirow[b]{2}{*}{ Costo Total } & \multicolumn{2}{|c|}{ Aportaciones } \\
\hline & & & & & Grupo & SAGARPA \\
\hline \multicolumn{7}{|l|}{ Inversion Fija } \\
\hline Vibrador recolector & unidad & 1.00 & $100,000.00$ & $100,000.00$ & 0.00 & $100,000.00$ \\
\hline Prelimpiadora & unidad & 1.00 & $150,000.00$ & $150,000.00$ & 0.00 & $150,000.00$ \\
\hline Seleccionadora & unidad & 1.00 & $240,000.00$ & $240,000.00$ & 0.00 & $240,000.00$ \\
\hline Desrueznador & unidad & 1.00 & $45,000.00$ & $45,000.00$ & 0.00 & $45,000.00$ \\
\hline Remolque & unidad & 1.00 & $7,000.00$ & $7,000.00$ & 7000.00 & 0.00 \\
\hline Arpillas & pza & $1,000.00$ & 0.80 & 800.00 & 800.00 & 0.00 \\
\hline Bascula & unidad & 1.00 & $1,000.00$ & $1,000.00$ & 1000.00 & 0.00 \\
\hline Tractor 2751 & unidad & 1.00 & $250,000.00$ & $250,000.00$ & 250000.00 & 0.00 \\
\hline Terreno & $\mathrm{Ha}$. & 0.50 & $100,000.00$ & $50,000.00$ & 50000.00 & 0.00 \\
\hline Subtotal & & & & - & - & 0.00 \\
\hline & & & & & & \\
\hline \multicolumn{7}{|l|}{ Capital de trabajo } \\
\hline Inventario & Unidad & 1.00 & $\$ 800$ & 800.00 & 800.00 & \\
\hline Costos Indirectos de fabricación & Unidad & 1.00 & $\$ 20,000$ & $20,000.00$ & $20,000.00$ & \\
\hline Subtotal & & & & $20,800.00$ & $20,800.00$ & - \\
\hline Gran Total & & & & $866,199.84$ & $329,600.00$ & $535,000.00$ \\
\hline Porcentaje de participacion & & & & $100 \%$ & $38.1 \%$ & $61.76 \%$ \\
\hline
\end{tabular}

Se concluye que el proyecto muestra datos referenciales que permiten manifestar su rentabilidad, solvencia para consolidarse y desarrollarse en el corto y mediano plazo, arrojando una VAN \$88,460, una TIR
(27.76\%) y una relación costo beneficio de 1.26, demostrando así una total factibilidad técnica y financiera.

La grafica 1 muestra que el $86.67 \%$ de los productores locales entrevistados están 
dispuestos a contratar un servicio que involucre la utilización de tecnología en el lugar de cultivo. La gráfica 2 muestra que el $76.67 \%$ de los productores locales entrevistados venden el producto después de la recolección, el método para la venta denominado "de campo", existiendo gran oportunidad para la empresa en términos de selección generando la posibilidad de obtener mayores ganancias así como también, una ganancia mayor para el productor.

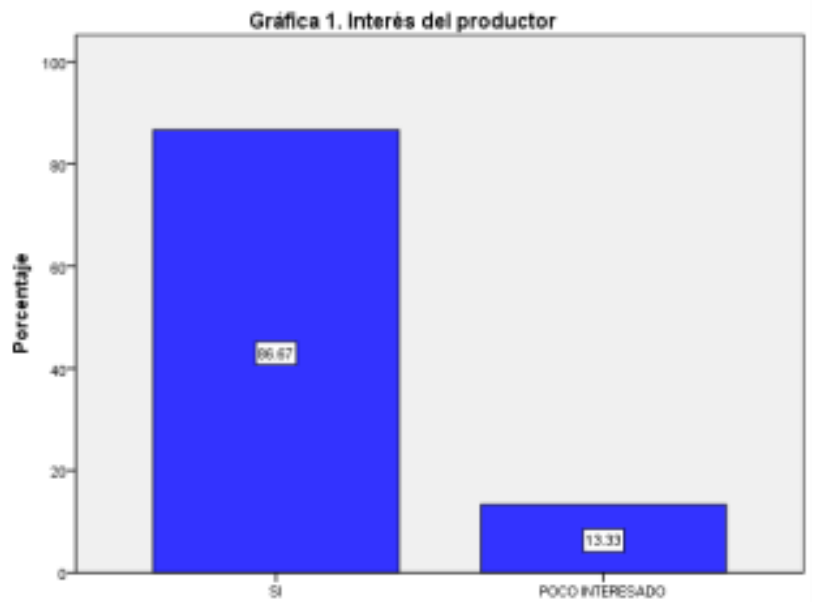

Interis del productor

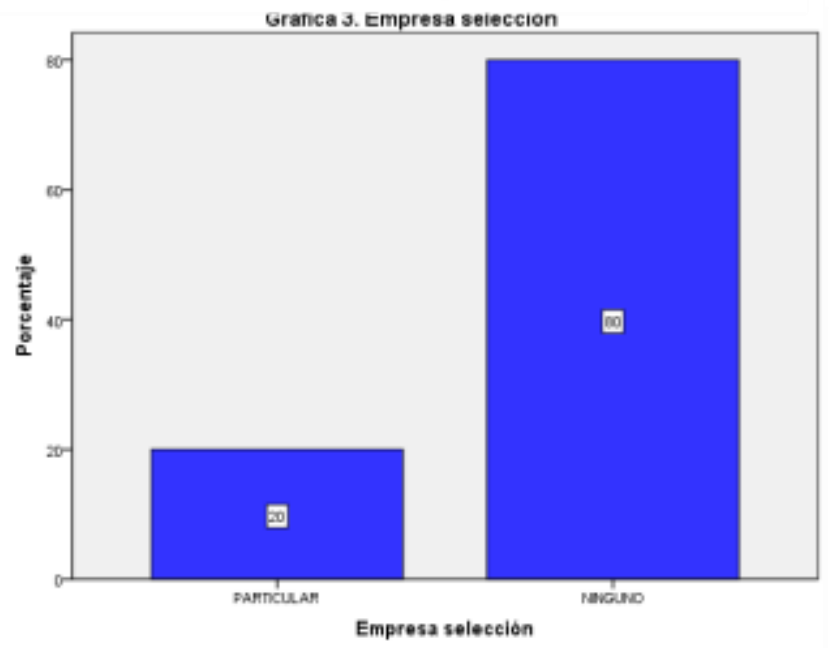

CONCLUSIONES

Con base en el estudio y la evaluación conducida, de las diferentes referencias
La grafica 3 muestra que el $80 \%$ de los productores locales entrevistados no realizan la selección a su producto. La grafica 4 muestra que el $30 \%$ de los productores locales entrevistados pagan un servicio adicional al cosechar el producto. Lo cual es un área potencial de oportunidad para el negocio y de este modo atraer mayor número de clientes.
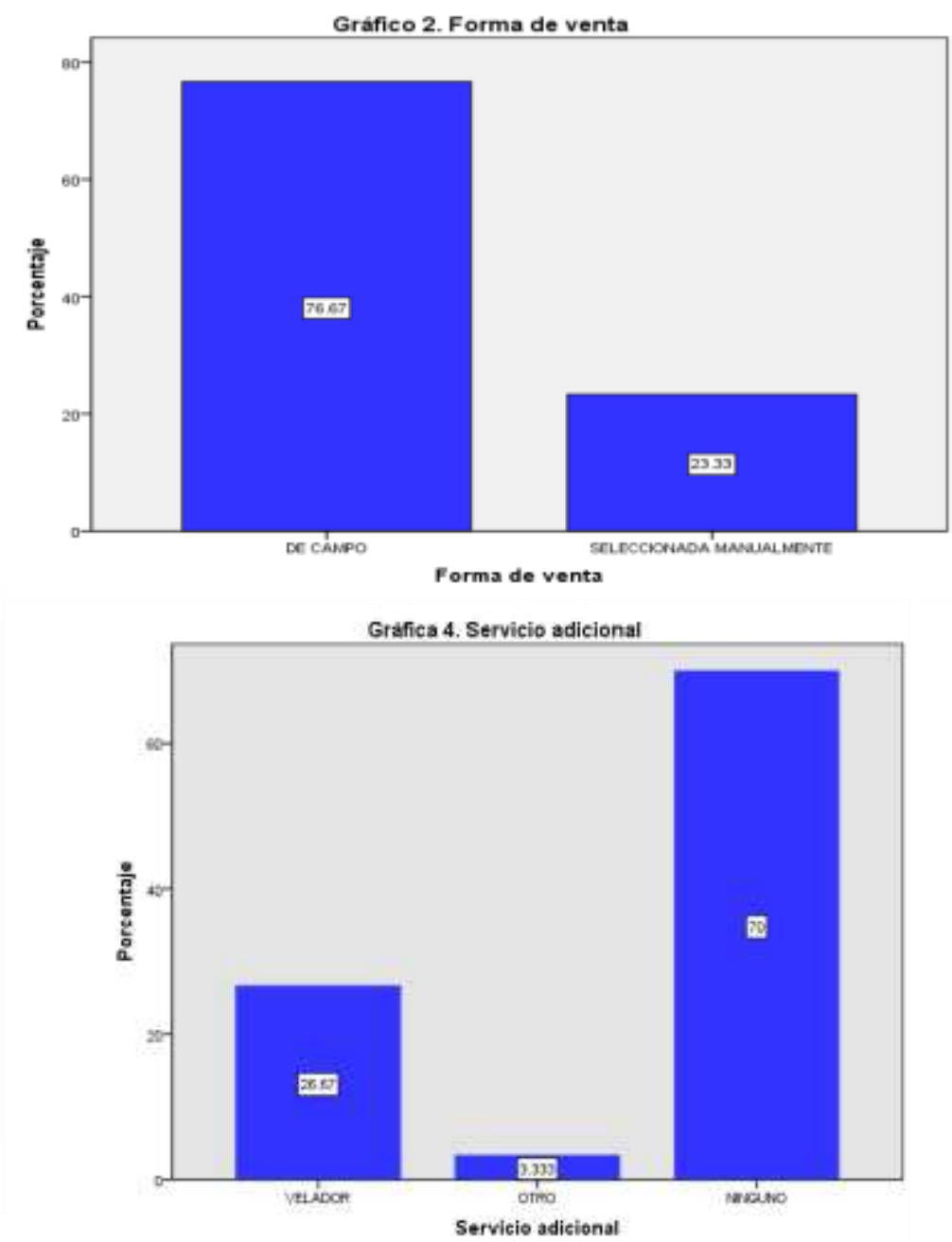

arrojadas en el ejercicio y en la elaboración del presente estudio se concluye de manera concreta lo siguiente: 
El proyecto de inversión elaborado muestra datos referenciales que permiten manifestar su rentabilidad basada principalmente en que los servicios que oferta la empresa tienen una aceptación del $86 \%$ de los productores locales encuestados. El proyecto es mercadológicamente, técnicamente y financieramente viable.Del mismo modo, el proyecto cuenta con toda la documentación necesaria para la obtención del recurso gubernamental, los cuales se presentarán en las fechas establecidas por los organismos correspondientes.

\section{LITERATURA CITADA}

Fuentes, F. Z. (2007). Creación de empresas agropecuarias. Morelia. México: Papiro Omega.
Stone, A. A., \& Gulvin, H. E. (1982). Maquinaria agrícola. México, D.F.: Continental.

SAGARPA. (s.f.). Recuperado el 17 de Febrero de 2015, de http://20062012.sagarpa.gob.mx/agricultura/prod uctodetemporada/_layouts/mobile/disp form.aspx.

SAGARPA. (s.f.). Recuperado el 18 de Febrero de 2015, de http://sagarpa.gob.mx/saladeprensa/20 12/Paginas/2014B019.aspx.

Financiera rural. (s.f.). Recuperado el 15 de Febrero de 2015, de http://www.financierarural.gob.mx/inf ormacionsectorrural/Panoramas/Panor ama\%20Nuez\%20(jul\%202014).pdf 
Copyright (c) 2018 José Eduardo Magaña Magaña, Victor Hugo Villarreal Ramirez, Maria Elvira González Anchondo,

Christian Mauricio Kiessling Davison y Abdón Palacios Monarrez

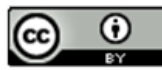

Este tex to está protegido por una licencia licencia Creative Commons 4.0

\begin{abstract}
Usted es libre para Compartir —copiar y redistribuir el material en cualquier medio o form ato- y Adaptar el documento -remezclar, transformar y crear a partir del material- para cualquier propósito,, incluso para fines comerciales, siempre que cumpla la condición de:

Atribución: Usted debe dar crédito a la obra original de manera adecuada, proporcionar un enlace a la licencia, e indicar si se han realizado cam bios. Puede hacerlo en cualquier form a razonable, pero no de form a tal que sugiera que tiene el apoyo del licenciante o 10 recibe por el uso que hace de la obra.

Resumendelicencia - Textocompletodelalicencia
\end{abstract}

